\title{
Indolent T cell lymphoproliferative disorder of the gastrointestinal tract: an uncommon case with lymph node involvement and the classic Hodgkin's lymphoma
}

\author{
Jie Wu ${ }^{1 \#}$, Long-Guang Li $^{2 \#}$, Xiang-Yan Zhang ${ }^{1}$, Li-Li Wang ${ }^{1}$, Li Zhang ${ }^{1}$, Yu-Jing Xiao ${ }^{1}$, Xiao-Ming Xing ${ }^{1}$, \\ Dong-Liang Lin ${ }^{1}$ \\ ${ }^{1}$ Department of Pathology, the Affiliated Hospital of Qingdao University, Qingdao, China; ${ }^{2}$ Center for Respiratory Pathology, State Key Lab of \\ Respiratory Disease, The First Affiliated Hospital, Guangzhou Medical University, Guangzhou, China \\ \#These authors contribute equally to this work. \\ Correspondence to: Dong-Liang Lin; Xiao-Ming Xing. Department of Pathology, the Affiliated Hospital of Qingdao University, No. 16, Jiangsu Road, \\ Qingdao, China. Email: lindongliang@outlook.com; edithxing@126.com.
}

\begin{abstract}
Here we describe an unusual case of an indolent CD8+ T-cell lymphoproliferative disorder in the gastrointestinal tract (ITCLD-GT) accompanied by neck lymph node infiltration and new onset of classic Hodgkin's lymphoma after two years follow-up. Previously, this 42-year-old Asian man suffered from diarrhea and abdominal pain for two years. Intestinal biopsies showed a group of small to intermediatesized lymphocytes which were monomorphic as well as arranged in a nodular pattern with no clear boundary and were diagnosed as ITCLD-GT. He did not receive chemotherapy or have any disease progression in the gastrointestinal tract (GIT) during the follow-up until a development of neck lymphadenopathy, which led to an eventual mixed cellularity type of Hodgkin's lymphoma, one type of classic HL diagnosis. Interestingly, besides the Hodgkin's cells, the same pathological T-cells in the GIT were present in the Hodgkin's lymphoma lesions. These two pathological T cells in GIT and neck lymph node had the identical histopathological and molecular abnormalities that confirmed the abenteric distant infiltration of ITCLDGT to the neck lymph node in this patient. This is the first case of ITCLD-GT that has a definite distant lymph node invasion. ITCLD-GT usually has a relatively good prognosis but patients with ITCLD-GT may have abenteric distant infiltration. Thus, long-term follow-up and further study of the underlying mechanisms of this process are necessary.
\end{abstract}

Keywords: Indolent T-cell lymphoproliferative disorder (ITCLD); gastrointestinal tract (GIT); Hodgkin's lymphoma; case report

Submitted Feb 03, 2020. Accepted for publication Jul 31, 2020.

doi: 10.21037/jgo-20-54

View this article at: http://dx.doi.org/10.21037/jgo-20-54

\section{Introduction}

Indolent T-cell lymphoproliferative disorders of the gastrointestinal tract (ITCLD-GTs) are rare lymphoid neoplasms that have been designated as a new disease entity in 2016 by the World Health Organization (1). Histopathological examination of the intestinal mucosa of patients with ITCLD-GT shows a partially preserved mucosa, in addition to chronic inflammation, glandular atrophy, and villous reduction. Small atypical cells of
T-lymphocyte origin can also be observed infiltrating the lamina propria or even submucosa. These cells have round or angulated nuclei with indistinct nucleoli. ITCLDGT lymphoepithelial lesions usually lack necrosis and do not demonstrate vascular invasion (2-4). Patients with this disease usually have a good prognosis although no standardized therapeutic regimen exists. Some patients have prolonged survival even with no drug treatment, with official recommendations calling for a careful 'watch and 

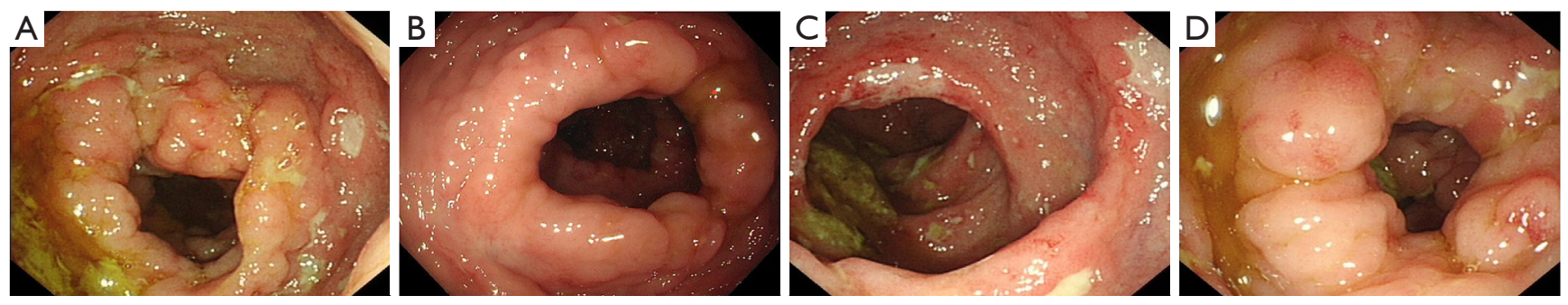

Figure 1 Endoscopic manifestation. (A,B) Electronic colonoscope examination in 2017 showed the multiple inflammatory bowel disease changes as rough hyperemic mucosa, multifocal deep ulcers but no masses. (C,D) Similar inflammation changes were found as rough hyperemic mucosa, multifocal deep ulcers in 2018 , with no mass too.

wait' approach (5-11). However, a few reports of ITCLDGTs showed its possibility of transformation to a highergrade lymphoma (12-15). In the paper by Perry et al. there were several patients who had enlarged region lymph nodes but little has been establish about whether ITCLD-GTs could infiltrate these lymph nodes because of the lack of pathological examinations (9). In this report we describe a unique, aggressive case of ITCLD-GT that is the first of our knowledge to have confirmed distant lymph node invasion as well as the incidence of a different type of lymphoma, Hodgkin's lymphoma. We present the following case series in accordance with the CARE-Guideline. Available at http://dx.doi.org/10.21037/jgo-20-54.

\section{Patient clinical history}

The patient was a 42-year-old man who presented a history of dental ulcers, abdominal pain, and diarrhea in September 2017. Bone marrow biopsy showed no abnormal cellular proliferative activity and a colonoscopy showed a rough hyperemic mucosa, multifocal deep ulcers suggestive of inflammatory bowel disease (IBD) changes in the rectum, sigmoid colon, descending colon, transverse colon, ascending colon, and cecum, with no observable masses (Figure $1 A, B)$. A biopsy of these lesions led to a diagnosis of ITCLD-GT. The patient was treated with mesalamine, compound glutamine, and the probiotic cocktail Bifico as a symptomatic and anti-inflammatory therapeutic regimen. However, no chemotherapy was given and the patient's response was limited. We identified neck lymphadenopathy on MRI during the patient's one-year ITCLD-GT follow up. A biopsy of the enlarged lymph nodes identified the same pathologic $\mathrm{T}$ cells found in the gastrointestinal tract (GIT) as well as classical cells associated with Hodgkin's lymphoma. Further endoscopic examination reappraised the gastrointestinal condition in October 2018 (Figure 1C,D), with similar inflammatory changes found the year prior. A biopsy showed the same pathological changes in the descending colon and cecum as in 2017, confirming that the diagnosis was still ITCLD-GT, yet there was no evidence of disease progression.

\section{Laboratory findings, pathological, immunophenotypic, and molecular findings}

Macroscopic examination showed similar GIT pathological features in 2017 and 2018. In 2017, multiple chronic inflammatory lesions were found in the ascending and sigmoid colon, as well as in the cecum. A predominant group of small to intermediate-sized lymphocytes was also noticed in the lamina propria (Figure 2). These lymphocytic cells were monomorphic and arranged in a nodular pattern with no clear boundary and no normal lymph node structures (e.g., follicles) were present. These cells also demonstrated an irregular nucleus with normally sized, yet pale cytoplasm. Mitotic figures were rare and no evidence of necrosis was present. The tumor cells seemed to be noninvasive and were restricted to the glands or crypts, with no observable lymphoepithelial lesions. Immunohistochemical (IHC) staining showed the pathologic lymphoid cells were positive for CD3, CD8, and TIA-1 and negative for CD20, CD4, CD5, CD56, and TdT. The proliferation index as determined by Ki-67 staining was $\sim 5-10 \%$ (Figure $2 B, C, D$ ). Molecular testing using a polymerase chain reaction (PCR) showed $\gamma$ TCR gene rearrangement (Figure 3). No immunoglobulin gene rearrangement was found. Histopathologic analysis in 2018 revealed similar morphological changes with no progression from the initial analysis in 2017 (Figure 2E). Repeat IHC again showed positive staining for $\mathrm{CD} 3, \mathrm{CD} 8$, and $\mathrm{CD} 43$ 

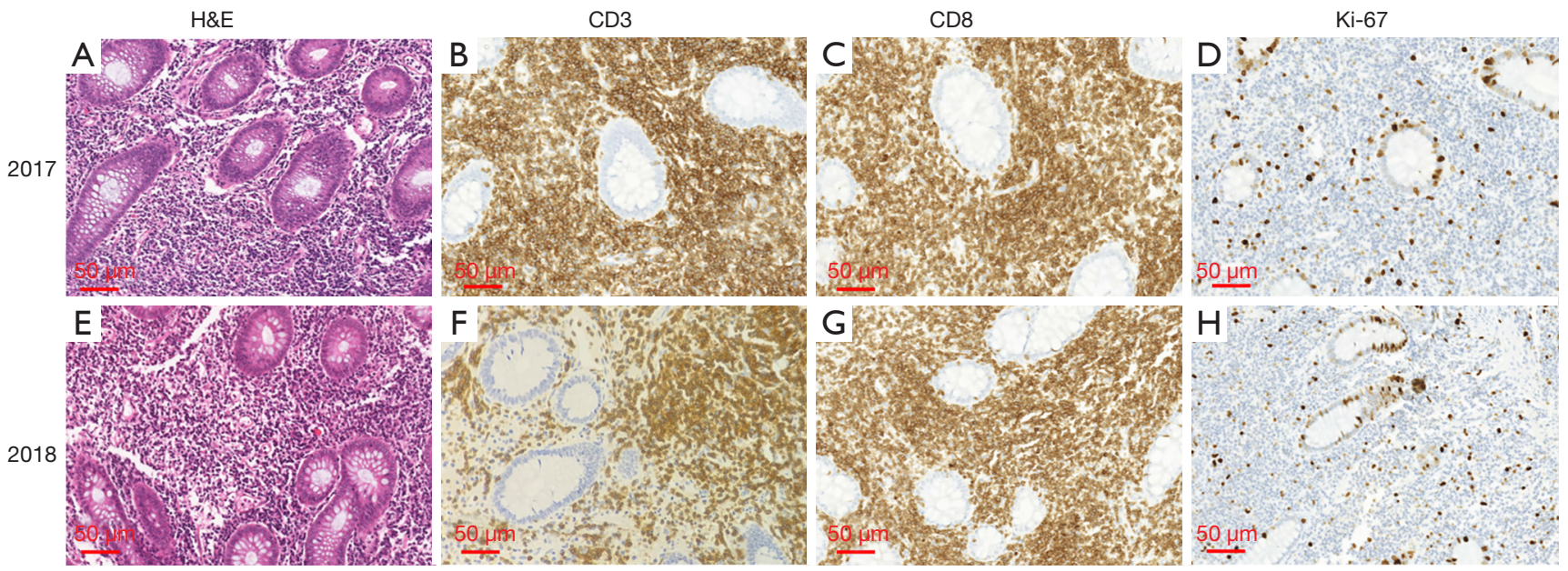

Figure 2 Histopathological changes in intestinal tract. (A,E) Microscopically, the lymphocytic cells were monomorphic and arranged with no clear boundary as well as no normal structure destroyed (H\&E staining; original magnification, ×200). (B,F) The lymphocytic cells were CD3 positive (immunohistochemistry staining; original magnification, $\times 200$ ). (C,G) The lymphocytic cells were CD8 positive (immunohistochemistry staining; original magnification, $\times 200)$. (D,H) The proliferation fraction as determined by Ki-67 was low (immunohistochemistry staining; original magnification, $\times 200$ ). H\&E, hematoxylin and eosin staining. (A,B,C,D) Showed the biopsy in 2017; (E,F,G,H) showed the biopsy in 2018.

(CD43 was not in the first analysis but was in this analysis) while negative for CD20, CD4 and fluorescence in situ hybridization for EBER (EBER was not in the first analysis but was in this analysis). An $8 \% \mathrm{Ki}-67$ proliferation was also similar to earlier analysis (Figure $2 F, G, H$ ).

During the 2018 follow-up, we also histologically evaluated the neck lymph nodes biopsy and found lesions consistent with the mixed cellularity type of Hodgkin's lymphoma. This included typical Hodgkin and ReedSternberg cells that were surrounded by lymphocytes, plasma cells, and eosinophilic granulocytes (Figure 4A). IHC of Hodgkin cells showed $\mathrm{CD}_{15} 5^{+}$and $\mathrm{CD} 30^{+}$cells that were also weakly positive for Pax-5 and CD20 (Figure $4 B, C, D$ ). In situ hybridization for EBER was also positive (Figure 4E). The background of the lesion was rich with small to medium-sized lymphocytes that were either $\mathrm{CD}^{+}$or $\mathrm{CD}^{+}$. The number of the $\mathrm{CD} 8^{+}$cells was much more than the $\mathrm{CD}^{+}$cells and the $\mathrm{CD} 8^{+}$cells were little larger than the $\mathrm{CD}^{+}$cells, which indicated the $\mathrm{CD} 8^{+}$cells were neoplastic (Figure 4F). The atypical CD8 ${ }^{+}$lymphocytes were similar to those in the GIT, and PCR testing showed they had overlapping peaks (Figure 3B). Antibodies used for IHC analysis are summarized in Table 1.

This study was approved by the Medical Ethics Committee of the affiliated hospital of Qingdao university. All procedures performed in this case study were in accordance with the ethical standards of the institutional and/or national research committee(s) and with the Helsinki Declaration (as revised in 2013). Written informed consent was obtained from the patient for publication of this Case report and any accompanying images. A copy of the written consent is available for review by the Editor-in-Chief of this journal.

\section{Discussion}

Mucosal T cells in the GIT are essential for both immunotolerance and immunoreaction (16). Proliferative disorders of these cells can result in diverse, benign or malignant clinical outcomes, necessitating the need for selection of an appropriate treatment modality (17). ITCLDGT is a rare but newly recognized disease entity, established during the 2016 revision of the World Health Organization's classification of lymphoid neoplasms (1). Our patient with ITCLD-GT had a clinical history and histopathologic disease features similar to prior reports $(5,6)$. The infiltrated atypical lymphoid cells were small to medium-sized and derived from $\mathrm{CD} 8^{+} \mathrm{T}$ cells. They also localized between the glands, had a low proliferative index, and showed no substantial disruption of the mucosal architecture. Benign morphologic changes such as the absence of lymphoepithelial lesions and necrosis help distinguish ITCLD-GT diagnosis 

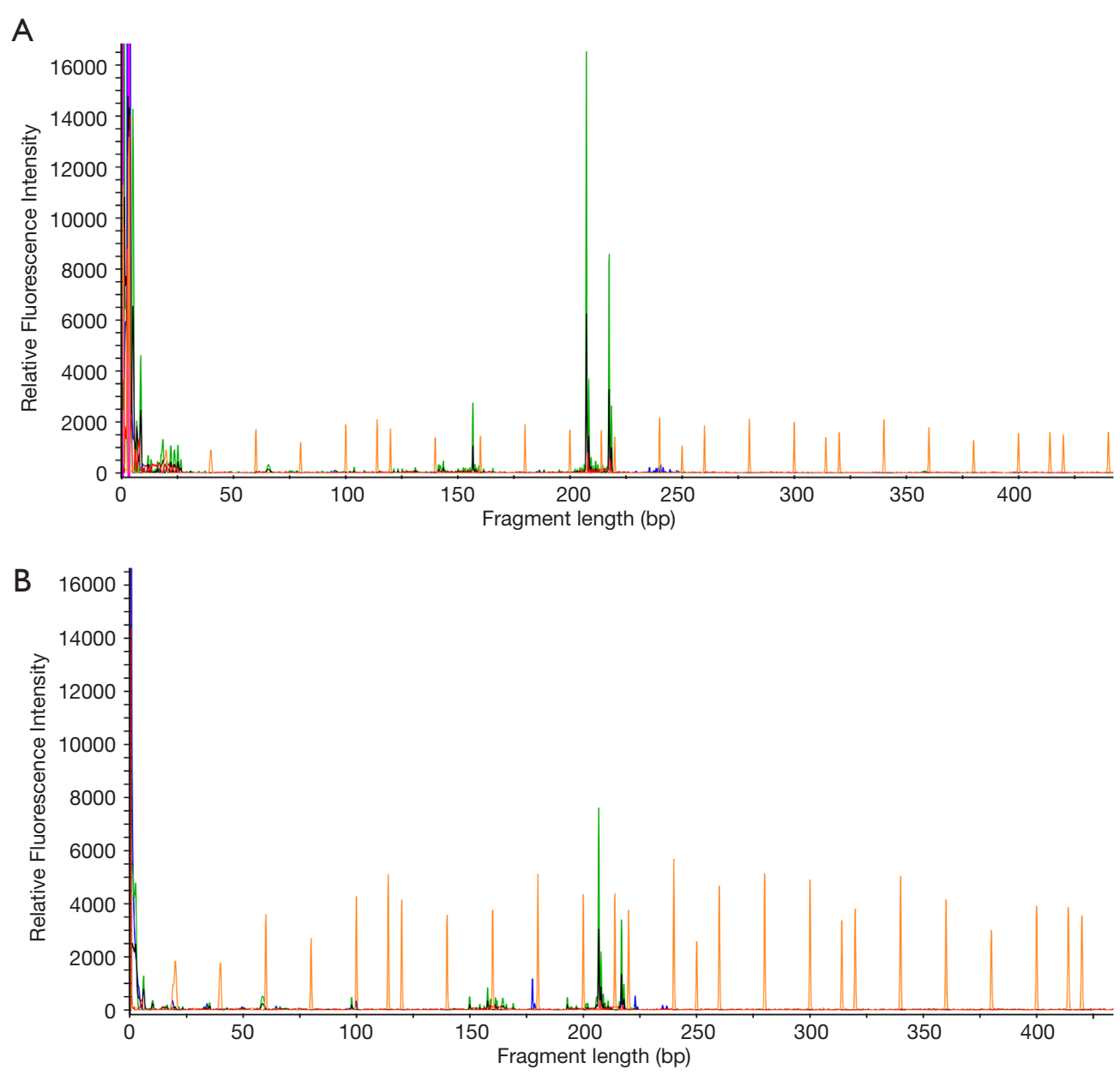

Figure 3 TCR gene rearrangement findings. (A) Molecular tests detected the TCR gene rearrangement by polymerase chain reaction (PCR) in intestinal biopsy. (B) Molecular tests detected the TCR gene rearrangement by polymerase chain reaction (PCR) in neck lymph nodes.

from other differential diagnoses, including IBD (which has lesions with more inflammatory features and enteropathyassociated T-cell lymphoma (EATL) which is highly invasive. Histopathology from the initial biopsy showed a group of atypical tumor-like cells that could have been easily misdiagnosed as type II EATL (18), however EATL is highly destructive with features of vessel infiltration and lymphoepithelial lesions, which was not found in our analysis $(19,20)$. Type II EATL is also CD $56^{+}$and CD43which is inconsistent with our patient's tumor. A low Ki-67 proliferation index in the 2017 and 2018 analysis confirmed the indolence of the tumor as well.

The most important characteristic of ITCLD-GT is its indolence, as the most often recommended strategy is a careful "watch and wait" approach. This is because chemotherapy is not recommended, as most patients have a good prognosis even without drug therapy (5-7). Prior reports suggest pathological cells usually remain in the GIT and are not likely to infiltrate distant sites (21). Lesion progression into a higher grade lymphoma has been reported but this conclusion remains controversial $(14,22)$. In our patient, the lesions initially appeared indolent during follow up with no evidence of a disease progression to a higher-grade lymphoma, suggesting the patient did not need to receive excessive medical treatment such as chemotherapy. Later follow up however revealed the new onset of an entirely distinct type and higher-grade lymphoma, at a distant location from the bowel, that was diagnosed as classic Hodgkin's lymphoma (mixed cellularity Hodgkin lymphoma, MCCHL). Analysis of the Hodgkin lesion in the neck revealed the same pathological lymphoid cells as the GIT. We confirmed the origin of these cells 

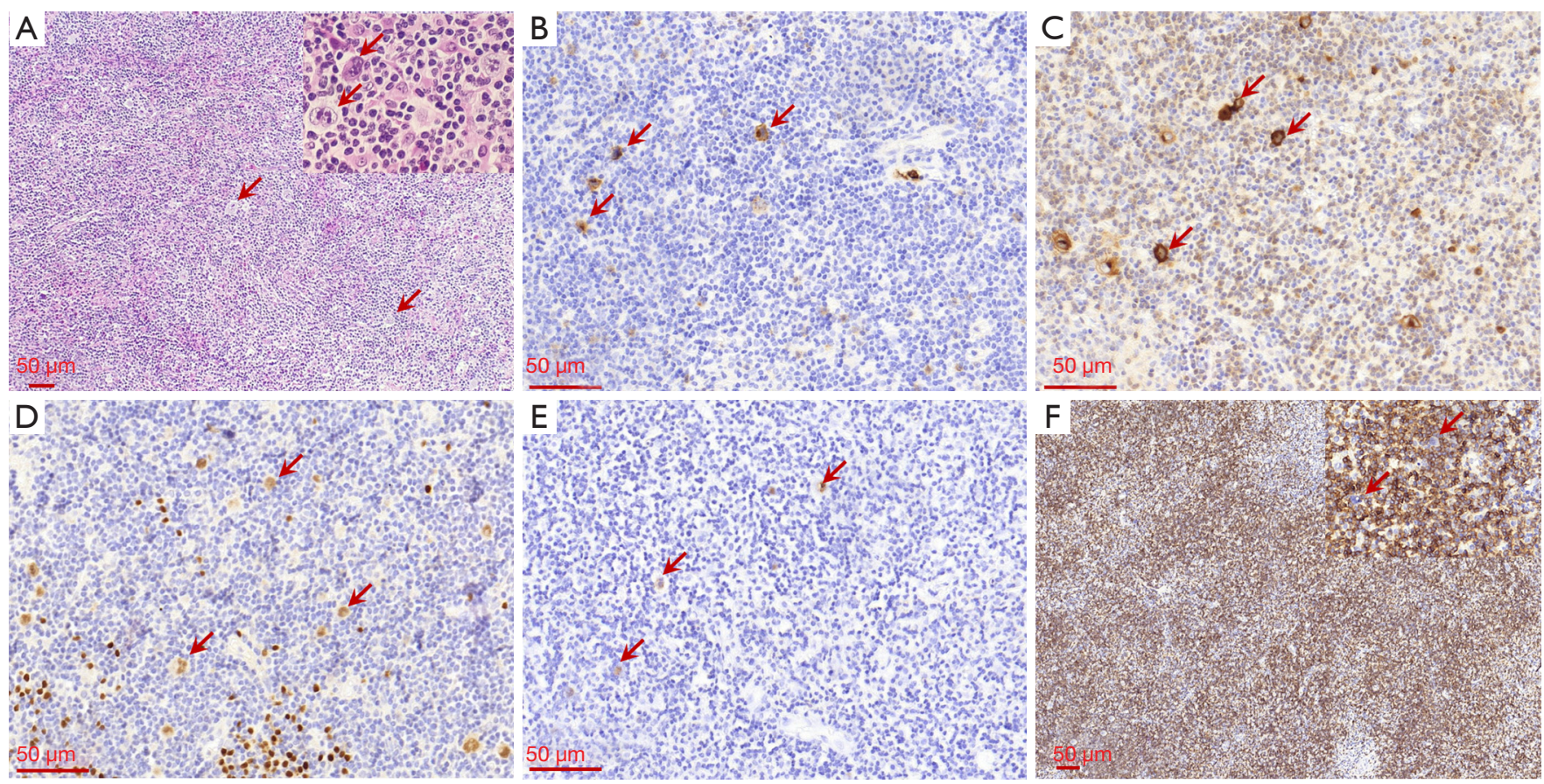

Figure 4 Histopathological findings in the lymph node lesion. (A) Microscopically, typical cells (indicated by the arrows) and lymphocytes, plasma cells, eosinophilic granulocytes were found (H\&E staining; original magnification, $\times 100)$, the HL cells were zoomed in. (B) The Hodgkin cells were CD15 positive (immunohistochemistry staining; original magnification, $\times 200$ ). (C) The Hodgkin cells were CD30 positive (immunohistochemistry staining; original magnification, $\times 200$ ). (D) The Hodgkin cells were Pax-5 week positive (immunohistochemistry staining; original magnification, $\times 200$ ). (E) EBER hybridization in situ showed the Hodgkin cells were positive (hybridization in situ staining; original magnification, $\times 200$ ). (F) Microscopically, the same pathological lymphocytic cells as in GIT were found (H\&E staining; original magnification, $\times 100$ ), the HL cells were zoomed in. In the background of the lymph node, the number of $\mathrm{CD}^{+}$cells were much more than the $\mathrm{CD}^{+}$ones and they were little larger than the $\mathrm{CD} 4^{+}$cells, which indicated the $\mathrm{CD} 8^{+}$cells were neoplastic. They are intermixed of the background.

Table 1 Immunohistochemistry antibodies

\begin{tabular}{llc}
\hline Antibody & Company & Catalog \\
\hline CD20 & Fuzhou Maixin Biotechnology, China & KIT0001 \\
CD5 & Fuzhou Maixin Biotechnology, China & KIT0033 \\
CD4 & Fuzhou Maixin Biotechnology, China & RMA0620 \\
CD56 & Fuzhou Maixin Biotechnology, China & MAB0743 \\
KI-67 & Fuzhou Maixin Biotechnology, China & MAB0672 \\
CD3 & Fuzhou Maixin Biotechnology, China & KIT0003 \\
CD8 & Fuzhou Maixin Biotechnology, China & RMA0514 \\
CD43 & Fuzhou Maixin Biotechnology, China & MAB0032 \\
CD15 & Fuzhou Maixin Biotechnology, China & MAB0779 \\
CD30 & Fuzhou Maixin Biotechnology, China & MAB0023 \\
PAX-5 & Roche, Germany & 790-4420 \\
EBER & Zhongshan Biotechnology, China & ISH-6021 \\
\hline
\end{tabular}

using analysis of cellular morphology, immunostaining, as well as genetic analysis. Our findings support the conclusion that that indolent, pathologic cells can distantly infiltrate other tissues, which is in agreement with a prior report on ITCLD-GT (21).

ITCLD-GT usually are Epstein-Barr virus (EBV) negative (22), most cases of $\mathrm{HL}$ are EBV negative too. However, the subtype of MCCHL is frequently associated with EBV (23-25). Our patient was EBV negative in the ITCLD-GT cells but positive in the Hodgkin cells. Prior work has shown that classic Hodgkin's lymphoma may begin to develop during viral infections, which induce an active immune response $(26,27)$, especially during acute EBV infections. Hodgkin's lymphoma cells are known to upregulate PD-L1, which can bind to inhibitory PD-1 receptors present on $\mathrm{T}$ cells (28). The PD-1/PD-L1 axis is also important in Hodgkin's lymphoma because genetic 
alterations such as 9p21.1 chromosomal rearrangement can mediate high PD-L1 expression. PD-L1 upregulation in Hodgkin's lymphoma following EBV infection is responsible for Hodgkin's lymphoma sensitivity to PD-1 blockade $(29,30)$. So, CHL genesis is related to immune downregulation of the body. In our case, the co-exist of $\mathrm{EBV}$ infection in the lymph node maybe an indispensable factor that trigger the onset of CHL.

In China, CHL is not very common accounting only for about $8 \%$ of all lymphomas, which is lower than in western countries. The case we report is quite unique because it covers two rare diseases. These two processes may completely unrelated, for example, given the persistent high burden of ITCLD-GTs in the bowel since 2017 , the neoplastic cells are probably circulating in the blood and some are homing into the neck lymph node. As we know, T cell accumulation in the Hodgkin's lymphoma tumor microenvironment as well as disparate $\mathrm{T}$ cell responses confirm that aberrant $\mathrm{T}$ cells with abnormal signal transduction may be critical for Hodgkin Lymphoma incident and enhancement (31,32). In our case, a subsequent CHL arising in a lymph node with involvement by ITCLD-GT, a group of aberrant T cells. Maybe, it still could not completely rule out that there is some potential unrevealed relationship between them which to be studied.

\section{Conclusions}

In summary, we report an unusual case of a CD8 ${ }^{+}$ITCLDGT with TCR $\gamma$ rearrangement which was accompanied by abenteric distant infiltration into neck lymph nodes and new onset of classic Hodgkin's lymphoma. To our knowledge, this is the first report of an ITCLD-GT with a newly acquired Hodgkin's lymphoma distantly. Notably the ITCLD-GT was found at multiple gastrointestinal sites yet no higher-grade lymphoma transformation occurred even though the patient was only given supportive therapy and no anti-neoplastic therapy. However, distant infiltration could happen as well as coexisted with other lesions. Thus, comprehensive evaluation of patients with ITCLD-GT is necessary for evaluation and selection of appropriate clinical treatments.

\section{Acknowledgments}

Funding: None.

\section{Footnote}

Reporting Checklist: The authors have completed the CARE reporting checklist. Available at http://dx.doi.org/10.21037/ jgo-20-54

Conflicts of Interest: All authors have completed the ICMJE uniform disclosure form (available at: http://dx.doi. org/10.21037/ jgo-20-54). The authors have no conflicts of interest to declare.

Ethical Statement: The authors are accountable for all aspects of the work in ensuring that questions related to the accuracy or integrity of any part of the work are appropriately investigated and resolved. This study was approved by the Medical Ethics Committee of the affiliated hospital of Qingdao university. All procedures performed in this case study were in accordance with the ethical standards of the institutional and/or national research committee(s) and with the Helsinki Declaration (as revised in 2013). Written informed consent was obtained from the patient for publication of this Case report and any accompanying images. A copy of the written consent is available for review by the Editor-in-Chief of this journal.

Open Access Statement: This is an Open Access article distributed in accordance with the Creative Commons Attribution-NonCommercial-NoDerivs 4.0 International License (CC BY-NC-ND 4.0), which permits the noncommercial replication and distribution of the article with the strict proviso that no changes or edits are made and the original work is properly cited (including links to both the formal publication through the relevant DOI and the license). See: https://creativecommons.org/licenses/by-nc-nd/4.0/.

\section{References}

1. Swerdlow SH, Campo E, Pileri SA, et al. The 2016 revision of the World Health Organization classification of lymphoid neoplasms. Blood 2016;127:2375-90.

2. Skinnider BF. Lymphoproliferative Disorders of the Gastrointestinal Tract. Arch Pathol Lab Med 2018;142:44-52.

3. Matnani R, Ganapathi KA, Lewis SK, et al. Indolent T- and NK-cell lymphoproliferative disorders of the gastrointestinal tract: a review and update. Hematol Oncol 2017;35:3-16. 
4. Said J. T-cell LPD of the GIT: first do no harm. Blood 2013;122:3548-50.

5. Soon G, Wang S. Indolent T-cell lymphoproliferative disease of the gastrointestinal tract in a renal transplant patient: diagnostic pitfalls and clinical challenges.

Pathology 2017;49:547-50.

6. Edison N, Belhanes-Peled H, Eitan Y, et al. Indolent T-cell lymphoproliferative disease of the gastrointestinal tract after treatment with adalimumab in resistant Crohn's colitis. Hum Pathol 2016;57:45-50.

7. Tabanelli V, Valli R, Righi S, et al. A unique case of an indolent myometrial T-cell lymphoproliferative disorder with phenotypic features resembling uterine CD8+ resident memory T cells. Pathobiology 2014;81:176-82.

8. Leventaki V, Manning JT Jr, Luthra R, et al. Indolent peripheral T-cell lymphoma involving the gastrointestinal tract. Hum Pathol 2014;45:421-6.

9. Perry AM, Warnke RA, Qinglong H, et al. Indolent T-cell lymphoproliferative disease of the gastrointestinal tract. Blood 2013;122:3599-606.

10. McElroy MK, Read WL, Harmon GS, et al. A unique case of an indolent CD56-positive T-cell lymphoproliferative disorder of the gastrointestinal tract: a lesion potentially misdiagnosed as natural killer/T-cell lymphoma. Ann Diagn Pathol 2011;15:370-5.

11. Ishitsuka K, Utsunomiya A, Aosaki S, et al. Indolent primary gastric adult T-cell leukemia/lymphoma with recurrent lesions limited to the stomach and duodenum. Rinshō ketsueki 2002;43:554-9.

12. Tanaka T, Megahed N, Takata K, et al. A case of lymphomatoid gastropathy: An indolent CD56-positive atypical gastric lymphoid proliferation, mimicking aggressive NK/T cell lymphomas. Pathol Res Pract 2011;207:786-9.

13. Guo L, Wen Z, Su X, et al. Indolent T-cell lymphoproliferative disease with synchronous diffuse large B-cell lymphoma: A case report. Medicine (Baltimore) 2019;98:e15323.

14. Perry AM, Bailey NG. Disease Progression in a Patient With Indolent T-Cell Lymphoproliferative Disease of the Gastrointestinal Tract. Int J Surg Pathol 2019;27:102-7.

15. Mneimneh WS, Shikhar Gautam V, Liang C, et al. Is ALK-gene rearrangement overlooked in primary gastrointestinal T-cell lymphomas? About two cases. Pathol Int 2015;65:666-70.

16. Konjar Š, Ferreira C, Blankenhaus B, et al. Intestinal Barrier Interactions with Specialized CD8 T Cells. Front Immunol 2017;8:1281.
17. Weindorf SC, Smith LB, Owens SR. Update on Gastrointestinal Lymphomas. Arch Pathol Lab Med 2018 142:1347-51.

18. Zing NPC, Fischer T, Zain J, et al. Peripheral T-Cell Lymphomas: Incorporating New Developments in Diagnostics, Prognostication, and Treatment Into Clinical Practice-PART 2: ENKTL, EATL, Indolent T-Cell LDP of the GI Tract, ATLL, and Hepatosplenic T-Cell Lymphoma. Oncology 2018;32:e83-9.

19. Ondrejka S, Jagadeesh D. Enteropathy-Associated T-Cell Lymphoma. Curr Hematol Malig Rep 2016;11:504-13.

20. Zhao L, Yang Y, Zhang Y. Type II enteropathy-associated t-cell lymphoma: A case report and literature review. Niger J Clin Pract 2018;21:812.

21. Wang X, Ng CS, Chen C, et al. An unusual case report of indolent T-cell lymphoproliferative disorder with aberrant CD20 expression involving the gastrointestinal tract and bone marrow. Diagn Pathol 2018;13:82.

22. Paulin MV, Couronne L, Beguin J, et al. Feline lowgrade alimentary lymphoma: an emerging entity and a potential animal model for human disease. BMC Vet Res 2018;14:306.

23. Daroontum T, Kohno K, Eladl AE, et al. Comparison of Epstein-Barr virus-positive mucocutaneous ulcer associated with treated lymphoma or methotrexate in Japan. Histopathology 2018;72:1115-27.

24. Vrzalikova K, Ibrahim M, Nagy E, et al. Co-Expression of the Epstein-Barr Virus-Encoded Latent Membrane Proteins and the Pathogenesis of Classic Hodgkin Lymphoma. Cancers (Basel) 2018;10:285.

25. Carbone A, Gloghini A, Caruso A, et al. The impact of EBV and HIV infection on the microenvironmental niche underlying Hodgkin lymphoma pathogenesis. Int J Cancer 2017;140:1233-45.

26. Merryman RW, Armand P, Wright KT, et al. Checkpoint blockade in Hodgkin and non-Hodgkin lymphoma. Blood Adv 2017;1:2643-54.

27. Carbone A, Gloghini A, Carlo-Stella C. Are EBV-related and EBV-unrelated Hodgkin lymphomas different with regard to susceptibility to checkpoint blockade? Blood 2018;132:17-22.

28. Vardhana S, Younes A. The immune microenvironment in Hodgkin lymphoma: T cells, B cells, and immune checkpoints. Haematologica 2016;101:794.

29. Menter T, Bodmer-Haecki A, Dirnhofer S, et al. Evaluation of the diagnostic and prognostic value of PDL1 expression in Hodgkin and B-cell lymphomas. Hum Pathol 2016;54:17-24. 
30. Dilly-Feldis M, Aladjidi N, Refait JK, et al. Expression of PD-1/PD-L1 in children's classical Hodgkin lymphomas. Pediatr Blood Cancer 2019;66:e27571.

31. Carreau NA, Diefenbach CS. Immune targeting of the microenvironment in classical Hodgkin's lymphoma: insights for the hematologist. Ther Adv Hematol

Cite this article as: Wu J, Li LG, Zhang XY, Wang L, Zhang LL, Xiao YJ, Xing XM, Lin DL. Indolent T cell lymphoproliferative disorder of the gastrointestinal tract: an uncommon case with lymph node involvement and the classic Hodgkin's lymphoma. J Gastrointest Oncol 2020;11(4):812819. doi: 10.21037/jgo-20-54
2019;10:2040620719846451.

32. Wörner S, Mueller-Hermelink HK, Voelker HU. Clinicopathologic features of adult EBV-associated B-cell lymphoproliferative disease. Pathol Res Pract 2018;214:207-12. 